\title{
Ivacaftor decreases monocyte sensitivity to interferon- $\gamma$ in people with cystic fibrosis
}

To the Editor:

Management of cystic fibrosis has been revolutionised by the introduction of cystic fibrosis transmembrane conductance regulator (CFTR) modulators. These compounds treat the underlying molecular basis of the disease by increasing activity of defective CFTR channels, which improves many clinical parameters and enhances patient quality of life [1]. Next-generation modulators, also known as triple combination therapy, promise to be highly efficacious in up to $90 \%$ of patients [2] and will likely dramatically change the landscape of cystic fibrosis disease. Studies examining individuals before and after initiation of CFTR modulators have revealed novel functions of CFTR and shown that CFTR modulators do not reverse all disease manifestations [3-5]. Thus, knowledge of the post-modulator cystic fibrosis disease state is crucial for understanding what continued therapies will be needed for people with cystic fibrosis and what new challenges may arise.

In prior work, we sought to identify immune pathways that were differentially modulated in the presence and absence of CFTR activity [6]. We isolated monocytes from subjects with G551D-CFTR mutations before and after initiation of the modulator ivacaftor. Using an unbiased proteomics approach, we identified post-ivacaftor changes in the monocyte plasma membrane proteome consistent with decreased interferon (IFN)- $\gamma$-related responses. Although both monocytes and macrophages from individuals with cystic fibrosis have been shown to manifest abnormal immune responses, myeloid IFN- $\gamma$ responses had not previously been reported as altered in people with cystic fibrosis [7]. In the current study, we took advantage of a second cohort of subjects with cystic fibrosis who were initiating ivacaftor to test the hypothesis that ivacaftor dampens monocyte sensitivity to IFN- $\gamma$.

We enrolled 10 patients, heterozygous for R117H-CFTR (5T) [8], from the Adult Cystic Fibrosis Clinic at St Vincent's University Hospital, Dublin, Ireland; second CFTR mutations were $\triangle \mathrm{F} 508$ ( $\mathrm{n}=7)$, M156R $(n=2)$ and $2662+1 \mathrm{G} \rightarrow A(n=1)$. The median age of subjects was 40.5 years (range $25-52$ years), six were male, median body mass index was $25.1 \mathrm{~kg} \cdot \mathrm{m}^{-2}$ (range $18.5-32.5 \mathrm{~kg} \cdot \mathrm{m}^{-2}$ ), median baseline sweat chloride was $79 \mathrm{mM}$ (range $61-98 \mathrm{mM}$ ) and median baseline forced expiratory volume in $1 \mathrm{~s}\left(\mathrm{FEV}_{1}\right)$ was $72 \%$ predicted (range 35-109\% predicted). Recent clinical sputum cultures were positive for Pseudomonas aeruginosa in two subjects and Staphylococcus aureus (with or without P. aeruginosa, Haemophilus or Achromobacter species) in five subjects. Three subjects had prior P. aeruginosa-positive cultures and one subject did not produce sputum. Patients were excluded if they had received ivacaftor within 6 months or if they had received antibiotics within 2 weeks of the day 0 visit. Patients were allowed to continue other standard cystic fibrosis therapies. Ivacaftor was initiated following the day 0 visit. No subject had cystic fibrosis-related diabetes. Seven of the subjects were pancreatic sufficient; three subjects were being evaluated for pancreatic insufficiency but had recent normal faecal elastase values. The Research Ethics Committee at St Vincent's University Hospital approved this study and all subjects provided written informed consent.

@ERSpublications

This study demonstrates that initiation of the CFTR modulator ivacaftor in people with cystic fibrosis and susceptible CFTR mutations causes an acute reduction in blood monocyte sensitivity to the key proinflammatory cytokine IFN- $\boldsymbol{\gamma}$ http://bit.ly/2TeI6LG

Cite this article as: Hisert KB, Birkland TP, Schoenfelt KQ, et al. Ivacaftor decreases monocyte sensitivity to interferon- $\gamma$ in people with cystic fibrosis. ERJ Open Res 2020; 6: 00318-2019 [https:// doi.org/10.1183/23120541.00318-2019].

Copyright $\odot$ ERS 2020. This article is open access and distributed under the terms of the Creative Commons Attribution NonCommercial Licence 4.0. 
All subjects experienced a pronounced decrease in sweat chloride between days 0 and 2 of ivacaftor (median decrease 25.5 mM, range 21-44 mM; $<<0.0001$ ), which persisted at day 7 ( $\mathrm{p}<0.0001$ compared to day 0 , data not shown). The cohort also experienced a statistically significant increase in $\mathrm{FEV}_{1}$ at day 2 (median 0.15 L, range 0.02-0.37 L; p=0.0022) and day 7 (median 0.19 L, range 0.05-0.69 L; $\mathrm{p}=0.0021$; data not shown). These findings confirmed that ivacaftor augmented CFTR activity in a clinically relevant manner within $48 \mathrm{~h}$, as seen previously in the G551D-CFTR cohort [5].

We purified monocytes from subjects' blood at days 0,2 and 7 of treatment, stimulated the cells in vitro with recombinant human IFN- $\gamma\left(100 \mathrm{ng} \cdot \mathrm{mL}^{-1}\right)$ and quantified IFN- $\gamma$ target gene expression. Monocytes isolated on day 0 mounted robust IFN- $\gamma$-induced transcription of IFN- $\gamma$-stimulated genes (ISGs); in contrast, monocytes isolated from the same subjects 7 days after starting ivacaftor had markedly decreased ISG expression in response to IFN- $\gamma$ stimulation (figure 1a). Monocytes obtained from a subset of subjects $(\mathrm{n}=6)$ at day 2 indicated that induction of ISGs was attenuated as early as $48 \mathrm{~h}$ post-ivacaftor (figure $1 \mathrm{~b}$ ). The transcriptional responses of pro-inflammatory genes not induced by IFN- $\gamma$, such as those encoding tumour necrosis factor and interleukin-6, were not changed following ivacaftor (data not shown).

CXCL10 GBP1

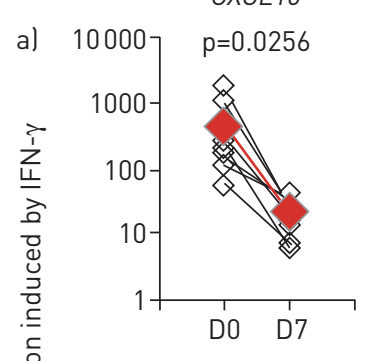

STAT1

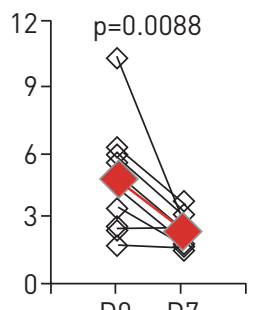

D0 D7

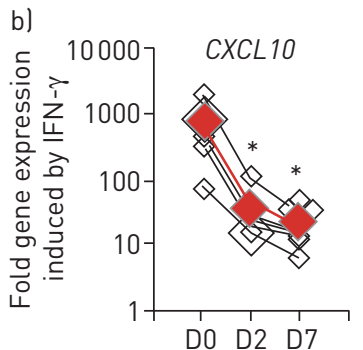

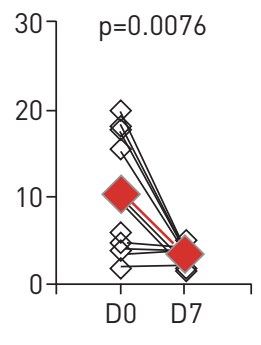

ICAM1
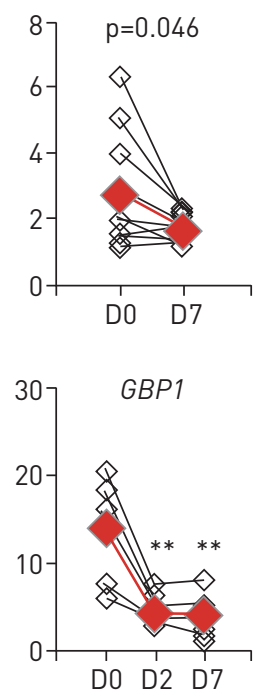

IRF1

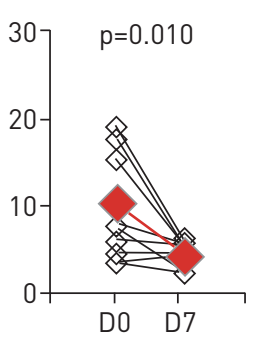

SOCS1
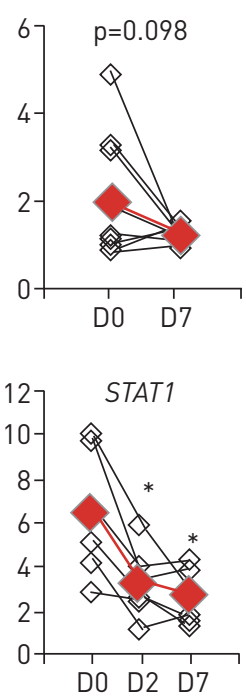
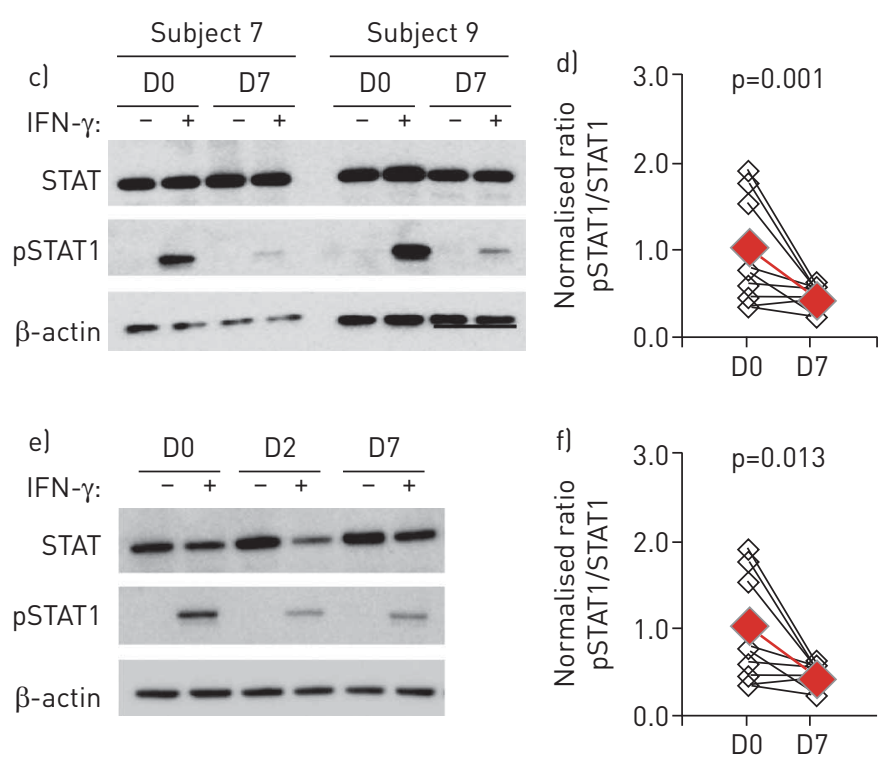

g)

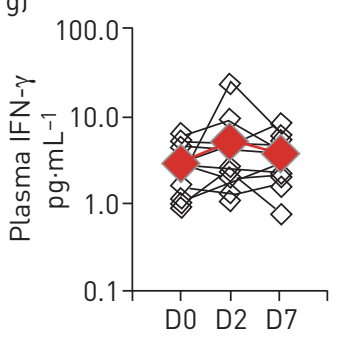

$\checkmark$ Individual subjects

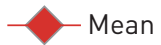

FIGURE 1 Decreased interferon (IFN)- $\gamma$-mediated responses in monocytes isolated from individuals with cystic fibrosis following initiation of ivacaftor therapy. a) Comparison of gene expression $3 \mathrm{~h}$ after stimulation ex vivo with IFN- $\gamma\left(100 \mathrm{ng} \cdot \mathrm{mL}^{-1}\right)$ in monocytes isolated at day D0 and D7 of ivacaftor treatment; HPRT was used as a housekeeping gene. $\mathrm{n}=10, \mathrm{p}$-values were calculated using Wilcoxon signed rank testing. Peripheral blood mononuclear cells were recovered from blood by density centrifugation and monocytes were isolated by CD14-positive selection magnetic bead separation (Miltenyi, Auburn, CA, USA). Monocytes were plated with or without recombinant human IFN- $\gamma$ (Peprotech, Rocky Hill, NJ, USA) in multiwell plates. Cells were lysed with RLT buffer (Qiagen, Valencia, CA, USA) for RNA analysis after $3 \mathrm{~h}$. RNA was isolated using the RNeasy Plus Kit (Qiagen) per manufacturer's instructions. Real-time reverse transcription PCR was performed on total RNA using TaqMan Gene Expression Assays primer/probe sets for target genes. b) Ivacaftor dampens monocyte responses to IFN- $\gamma$ within 2 days of starting therapy; $n=6$. *: $p<0.05$; **: $p \leqslant 0.005$ by ANOVA. c-f) Monocytes isolated from subjects at specified time points after initiation of ivacaftor were exposed to IFN- $\gamma$ for $1 \mathrm{~h}$ then lysed with Laemmli buffer (Alfa Aesar, Haverhill, MA, USA). Immunoblots were performed to determine abundance of STAT1 and phosphorylated STAT1 (pSTAT1); $\beta$-actin was used as a housekeeping control protein. c) Representative immunoblots from two subjects showing results from D0 and D7 lysates. d) Densitometry was performed to quantitate changes in pSTAT abundance; $n=8, p$-value calculated using Wilcoxon signed rank testing. e) Representative immunoblots from subject 3 showing results from D0, D2 and D7. f) Densitometry comparing abundance of pSTAT at day 2 versus day $0 ; n=4, p$-value calculated using Student's t-test. g) Ivacaftor did not cause changes in plasma IFN- $\gamma$ concentrations during the first week of treatment, as measured by Meso Scale Discovery biomarker assay. Statistical comparisons noted above were performed using Excel (Student's t-test) or GraphPad Prism 7 (Wilcoxon signed rank testing and ANOVA); $p<0.05$ was considered significant. 
IFN- $\gamma$ signalling is mediated by the JAK-STAT pathway [9]: the IFN- $\gamma$ receptor activates JAK proteins resulting in phosphorylation of STAT1, which translocates to the nucleus and increases transcription of ISGs. STAT1 protein levels were similar in monocytes recovered before and after initiation of ivacaftor (figure 1c). However, consistent with the decrease in ISG expression, IFN- $\gamma$-induced STAT1 phosphorylation was reduced in monocytes recovered on day 7 compared to monocytes isolated before initiation of ivacaftor (figure 1c and d). Decreased phosphorylation of STAT1 in response to IFN- $\gamma$ exposure was detected within $48 \mathrm{~h}$ of subjects beginning ivacaftor (figure 1e and $\mathrm{f}$ ). Because monocytes can become either primed by or tolerant to IFN- $\gamma$ based on prior exposure to differential doses of IFN- $\gamma$ [10], we also evaluated plasma IFN- $\gamma$ levels. Plasma IFN- $\gamma$ concentrations were unchanged during the first week of ivacaftor (figure $1 \mathrm{~g}$ ), consistent with analyses from our previous cohort [6].

These data reveal that ivacaftor causes a reduction in acute responsiveness of peripheral blood monocytes to IFN- $\gamma$ in people with cystic fibrosis, a change that may be clinically relevant. However, because our experiments used a single (likely supraphysiological) dose of IFN- $\gamma$, the clinical relevance of our findings remains unclear. Another limitation of this study is that we tested only one timepoint following cell stimulation with IFN- $\gamma$, and thus the data do not differentiate between a decreased amplitude of response to IFN- $\gamma$ or a delayed response. A third limitation is that this study does not demonstrate the mechanism by which ivacaftor decreases monocyte IFN- $\gamma$ sensitivity. Ivacaftor may be acting by a CFTR-dependent mechanism, as has been described for other cystic fibrosis-related myeloid cell defects $[11,12]$. Ivacaftor could also act directly on monocytes by a CFTR-independent mechanism; recent studies observed that CFTR modulators may have immune-dampening effects on macrophages that lack modulator-susceptible CFTR mutations [12, 13]. Finally, reduced monocyte IFN- $\gamma$ responses could be a secondary effect of ivacaftor. Prior work suggests that cystic fibrosis monocytes are impaired secondary to changes in plasma mediators caused by chronic airway infection [14]; thus, ivacaftor-induced changes in airway bacterial burden and inflammatory cytokines [5] could alter plasma immune factors. In this current cohort, as in our prior cohort [6], we did not detect changes in numbers of peripheral blood mononuclear cells, monocytes, T-cells or B-cells 1 week after initiation of ivacaftor (data not shown); however, ivacaftor may produce other alterations in systemic inflammation not detected by these assays.

Studying primary cells from subjects with cystic fibrosis provides the most clinically relevant characterisation of changes in immune cells after initiation of CFTR modulators; however, this study design produces results that are often challenging to recreate in vitro. Negative results from in vitro studies (e.g. addition of ivacaftor or CFTR inhibitors to freshly isolated monocytes) suggest a lack of direct effect of ivacaftor or CFTR activity on monocyte IFN- $\gamma$ responses. Yet, because monocytes have rapid turnover in the blood [15], it remains possible that the influence of ivacaftor or CFTR activity on monocyte responses occurs during haematopoiesis and cannot be reproduced in vitro using mature peripheral blood monocytes. Even without a definitive mechanism for how ivacaftor changes monocyte sensitivity to IFN- $\gamma$, these findings have intriguing and potentially clinically significant implications, as IFN- $\gamma$ plays a central role in modulating immune functions and promoting antimicrobial activities [9]. Changes in monocyte IFN- $\gamma$ responses may contribute to ivacaftor-induced decreases in airway bacterial burden and inflammation [5]. Exuberant myeloid cell IFN- $\gamma$-related responses could contribute to pathologic cystic fibrosis airway inflammation, and a reduction in these responses could shift monocytes and macrophages to less inflammatory phenotypes. Alternately, IFN- $\gamma$ can arrest monocyte migration [16] and dampening of IFN- $\gamma$ responses could enhance trafficking of pathogen-fighting monocytes to the cystic fibrosis airway. IFN- $\gamma$ is also important for upregulation of pathogen receptors, as well as antigen processing and presentation, especially of intracellular bacteria such as mycobacterial species [9]; thus, dampening IFN- $\gamma$ responses could have unexpected negative consequences, causing individuals with cystic fibrosis to become more susceptible to these pathogens. In fact, prior studies of T-cell responses in cystic fibrosis suggested that the cystic fibrosis airway could be an IFN- $\gamma$-deficient environment $[17,18]$, leading to a clinical trial of nebulised IFN- $\gamma$ in people with cystic fibrosis [19]. This study was halted early due to an increase in exacerbations in the treatment group, highlighting the fact that IFN- $\gamma$ responses are complex and their role in cystic fibrosis requires further elucidation. Follow-up studies are needed to clarify the contributions of monocyte responses to cystic fibrosis airway inflammation and how initiation of CFTR modulators will modify immune responses in the cystic fibrosis lung.

Katherine B. Hisert $\oplus^{1}$, Timothy P. Birkland ${ }^{1}$, Kelly Q. Schoenfelt ${ }^{2}$, Matthew E. Long ${ }^{1}$, Brenda Grogan ${ }^{3}$, Suzanne Carter ${ }^{3}$, W. Conrad Liles ${ }^{1}$, Edward F. McKone ${ }^{3}$, Lev Becker ${ }^{2,4}$ and Anne M. Manicone $\mathbf{1}^{1,4}$

${ }^{1}$ Dept of Medicine, University of Washington, Seattle, WA, USA. ${ }^{2}$ Ben May Dept for Cancer Research, University of Chicago, Chicago, IL, USA. ${ }^{3}$ Dept of Medicine, St Vincent's University Hospital, Dublin, Ireland. ${ }^{4}$ These authors contributed equally. 
Correspondence: Katherine Hisert, National Jewish Health, 1400 Jackson St, Smith A550, Denver, CO 80206, USA. E-mail: hisertk@njhealth.org

Received: 03 Dec 2019 | Accepted after revision: 18 Feb 2020

Acknowledgements: We thank Pradeep Singh (University of Washington, Seattle, WA, USA) for inviting us to join the research team in Dublin, Ireland; we also thank Michael Welsh (University of Iowa, Iowa City, IA, USA), David Stoltz (University of Iowa), Jan Launspach (University of Iowa), Mavra Nasir (Dartmouth University, Hanover, NH, USA), Anina Ratjen (University of Washington), Robert Gray (University of Edinburgh, Edinburgh, UK) and Gareth Hardisty (University of Edinburgh) for collegial interactions and strategic support during specimen collection and initial specimen processing in Dublin.

Author contributions: K.B. Hisert, E.F. McKone and L. Becker conceived the hypothesis and designed the study. K.B. Hisert, T.P. Birkland, K.Q. Schoenfelt, B. Grogan, S. Carter, E.F. McKone, L. Becker and A.M. Manicone acquired and analysed the data. K.B. Hisert, M.E. Long, W.C. Liles, L. Becker and A.M. Manicone prepared the manuscript.

Support statement: This work was funded by a CF Research Innovation Award from Vertex Pharmaceuticals, Inc. Funding information for this article has been deposited with the Crossref Funder Registry.

Conflict of interest: K.B. Hisert reports a CF Research Innovation Award from Vertex Pharmaceuticals during the conduct of the study. T.P. Birkland has nothing to disclose. K.Q. Schoenfelt has nothing to disclose. M.E. Long has nothing to disclose. B. Grogan has nothing to disclose. S. Carter reports grants from the Irish Thoracic Society, Gilead and the Cystic Fibrosis Association of Ireland during the conduct of the study. W.C. Liles has nothing to disclose E.F. McKone reports a grant from Vertex Pharmaceuticals during the conduct of the study; and advisory boards fees from Vertex Pharmaceuticals and Proteostasis, and speaker fees from Roche and Novartis, outside the submitted work. L. Becker has nothing to disclose. A.M. Manicone has nothing to disclose.

\section{References}

1 Heltshe SL, Cogen J, Ramos KJ, et al. Cystic fibrosis: the dawn of a new therapeutic era. Am J Respir Crit Care Med 2017; 195: 979-984.

2 Taylor-Cousar JL, Mall MA, Ramsey BW, et al. Clinical development of triple-combination CFTR modulators for cystic fibrosis patients with one or two F508del alleles. ERJ Open Res 2019; 5: 00082-2019.

3 Adam RJ, Hisert KB, Dodd JD, et al. Acute administration of ivacaftor to people with cystic fibrosis and a mutation reveals smooth muscle abnormalities. JCI Insight 2016; 1: e86183.

4 Rowe SM, Heltshe SL, Gonska T, et al. Clinical mechanism of the cystic fibrosis transmembrane conductance regulator potentiator ivacaftor in G551D-mediated cystic fibrosis. Am J Respir Crit Care Med 2014; 190: 175-184.

5 Hisert KB, Heltshe SL, Pope C, et al. Restoring CFTR function reduces airway bacteria and inflammation in people with cystic fibrosis and chronic lung infections. Am J Respir Crit Care Med 2017; 195: 1617-1628.

6 Hisert KB, Schoenfelt KQ, Cooke G, et al. Ivacaftor-induced proteomic changes suggest monocyte defects may contribute to the pathogenesis of cystic fibrosis. Am J Respir Cell Mol Biol 2016; 54: 594-597.

7 Bruscia EM, Bonfield TL. Cystic fibrosis lung immunity: the role of the macrophage. J Innate Immun 2016; 8 : 550-563.

8 Chen N, Prada AE. Development of allele-specific multiplex PCR to determine the length of poly-T in intron 8 of CFTR. PeerJ 2014; 2: e468.

9 Schroder K, Hertzog PJ, Ravasi T, et al. Interferon-gamma: an overview of signals, mechanisms and functions. J Leukoc Biol 2004; 75: 163-189.

$10 \mathrm{Hu}$ X, Herrero C, Li WP, et al. Sensitization of IFN-gamma Jak-STAT signaling during macrophage activation. Nat Immunol 2002; 3: 859-866.

11 Di Pietro C, Zhang PX, O’Rourke TK, et al. Ezrin links CFTR to TLR4 signaling to orchestrate anti-bacterial immune response in macrophages. Sci Rep 2017; 7: 10882.

12 Zhang S, Shrestha CL, Kopp BT. Cystic fibrosis transmembrane conductance regulator (CFTR) modulators have differential effects on cystic fibrosis macrophage function. Sci Rep 2018; 8: 17066.

13 Barnaby R, Koeppen K, Nymon A, et al. Lumacaftor (VX-809) restores the ability of CF macrophages to phagocytose and kill Pseudomonas aeruginosa. Am J Physiol Lung Cell Mol Physiol 2018; 314: L432-LL38.

14 del Fresno C, Gomez-Pina V, Lores V, et al. Monocytes from cystic fibrosis patients are locked in an LPS tolerance state: down-regulation of TREM-1 as putative underlying mechanism. PLoS One 2008; 3: e2667.

15 Patel AA, Zhang Y, Fullerton JN, et al. The fate and lifespan of human monocyte subsets in steady state and systemic inflammation. J Exp Med 2017; 214: 1913-1923.

$16 \mathrm{Hu} \mathrm{Y,} \mathrm{Hu} \mathrm{X,} \mathrm{Boumsell} \mathrm{L,} \mathrm{et} \mathrm{al.} \mathrm{IFN-gamma} \mathrm{and} \mathrm{STAT1} \mathrm{arrest} \mathrm{monocyte} \mathrm{migration} \mathrm{and} \mathrm{modulate} \mathrm{RAC/CDC42}$ pathways. J Immunol 2008; 180: 8057-8065.

17 Moss RB, Hsu YP, Olds L. Cytokine dysregulation in activated cystic fibrosis (CF) peripheral lymphocytes. Clin Exp Immunol 2000; 120: 518-525.

18 Tiringer K, Treis A, Fucik P, et al. A Th17- and Th2-skewed cytokine profile in cystic fibrosis lungs represents a potential risk factor for Pseudomonas aeruginosa infection. Am J Respir Crit Care Med 2013; 187: 621-629.

19 Moss RB, Mayer-Hamblett N, Wagener J, et al. Randomized, double-blind, placebo-controlled, dose-escalating study of aerosolized interferon gamma- $1 \mathrm{~b}$ in patients with mild to moderate cystic fibrosis lung disease. Pediatr Pulmonol 2005; 39: 209-218. 